\title{
Studies on the Agaricaceae of Japan I. Volvate Agarics in Hokkaido."
}

$\mathrm{By}$

\section{Sanshi Imai")}

Received March 2\%, 1993

In the course of the study on the Agaricaceae of Japan, the writer obtained a considerable number of specimens of the volvate agarics. Among them, the species collected in Hokkaido are selected and preliminarily reported in this short paper.

Amanita (Fr. ex Pers.) QuéL.

Champ. Jura Vosg. 1, 23, 1872.

Amanita Pers. Syn. Fung. 246, 1801.

Agaricus $\S$ Amanita Fr. Syst. Myc. 1, 12, 1821.

Venenarius EarLe, Bull. N. Y. Bot. Mus. j, 450, 1909.

Leucomyces EARLE, ibid. 451, 1909.

Sect. Volvatre Schrokt. Pilze Schles. 1, 680, 1889.

1. Amanita Caesarea (Fr. ex Scop.) QuéL.

Champ. Jura Vosg. 1, 24, 1872.

Agaricus Caesareus Scop. Fl. Carn. ed. 2, 2, 419, 1772.

Amanita caesarea Pers. Syn. Fung. 252, 1801.

Amanita aurantiaca Pers. ibid. 252, 1801.

Agaricus (Amanita) Caesareus Fr. Syst. Myc. 1, 15, 1821.

Amanita pellucida Banv. et Pk. in Pr. Ann. Rep. N. Y. St. Mus. 44, 178, 1892.

Venenarius caesareus MurriLl, Mycologia $\check{5}, 73,1913$.

Hab. On the ground in woods, in solitary or scattered. Sept.-Oct.

Distr. Hokkaido (Ishikari, Iburi \& Kushiro) \& Honshu. Europe \& North America.

Jap. name. Tamago-take, Ô-benitake. (卵咠, 大紅咠)

1) The present paper was prepared to read at the meeting of the Botanical Society of Japan on April 1, 1933.

2) The writer wishes to express his sincere thanks to Prof. Emer. K. Mirabe and Prof. S. ITo for their many kind advices in various ways. 


\section{Amanita Phalloides (Fr.) QuéL.}

Champ. Jura Vosg. 1, 25, 1872.

Agaricus (Amanita) Phalloides Fr. Syst. Myc. 1, 13, 1821.

Venenarius phalloides MurriLt, Mycologia 4, 240, 1912.

Hab. On the ground in woods, in solitary or scattered. Aug.-Oct.

Distr. Hokkaido (Ishikari) \& Honshu. Europe \& North·America. Jap. name. Tamago-ten gutake. (卵天狗茸).

3. Amanita verna ( $F_{R}$. ex Bull.) GiLl.

Hymén. Fr. 37, 1874.

Agaricus bulbosus vernus BulL. Champ. Fr. pl. 108, 1782.

Amanita verna Pers. Syn. Fung. 250, 1801.

Agaricus (Amanita) vernus Fr. Syst. Myc. 1, 13, 1821; Epicr. 4, 1836.

Hab. On the ground in woods, in solitary or scattered. Aug.Sept.

Distr. Hokkaido (Ishikari \& Iburi) \& Honshu. Europe, North America \& Australia.

Jap. name. Shiro-tamago-tengutake. (白卵天狗咠).

4. Amanita virosa (Fr.) Quét.

Champ. Jura Vosg. 1, 24, 1872.

Agaricus (Amanita) virosus Fr. Epicr. 3, 1836.

Hab. On the ground in woods, in solitary or scattered. Sept.

Distr. Hokkaido (Ishikari) \& Honshu. Europe.

Jap. name. Doku-tsuru-take. (毒鶴茸).

5. Amanita subjunquillea Imar, sp. nov.

Pileo 3-7 cm. lato, conico-convexo, dein subplano, laevi, glabro, viscidulo, flavo vel flavo-ochraceo, margine pallidiore raro striatuloque, fragmentis volvae saepe squamoso; carne alba, tenui, odore saporeque nullo ; lamellis liberis, albis, subconfertis ; stipite $6-11 \mathrm{~cm}$. longo, 6-10 mm. crasso, sursum attenuato vel subaequali, deorsum subbulboso, albido vel flavidulo, flavo- vel brunneo-fibrilloso-squamuloso, subsolido ; annulo apicali, membranaceo, tenui, persistante, albo striatoque, subtus flavidulo; volva alba vel brunneola, vaginali, limbo 2-3 lobata, membranacea; sporis globosis vel subglobosis, $7-8 \mu$ vel $7.5 \times 7 \mu$, hyalinis.

Hab. On the ground in woods, in solitary or scattered. Sept.

Distr. Hokkaido (Ishikari).

Jap. name. Tamago-take-modoki. (擬卵茸).

The present fungus resembles Amanita junquillea QuÉL. which was 
described as the spores are spherical ovoid, but the latter has been now accepted as an identical species with Amanitopsis adnata, Amanitopsis gemmata or Amanita russuloides. The fungus under consideration is easily distinguished from above mentioned species by the stem which is decorated with the yellowish or brownish small fibrous scales and has a distinct apical ring.

6. Amanita porphyria (Fr. ex Alb. et Schw.) Gill. Hymén. Fr. 35, 1874.

Amanita porphyria Arb. et Schw. Consp. Fung. 142, 1805. Agaricus (Amanita) porphyrius Fk. Syst. Myc. 1, 14, 1821. Venenarius porphyrius MURriLL, Mycologia 5, 81, 1913. Hab. On the ground in woods, in solitary or scattered. Oct. Distr. Hokkaido (Ishikari) \& Honshu. Europe \& North America. Jap. name. Ko-tengu-take. (小天狗咠).

7. Amanita Mappa (Fr. ex Batsch) QuéL. Champ. Jura Vosg. 1, 25, 1872.

Agaricus Mappa BAtsch, El. Fung. 57, 1783. Amanita citrina $\beta$ Mappa Pers. Syn. Fung. 251, 1801, Agaricus (Amanita) Mappa Fr. Epicr. 6, 1836.

Hab. On the ground in woods, in solitary or gregarious. Sept.-Oct. Distr. Hokkaido (Ishikari) \& Honshu. Europe, North America \& Australia.

Jap. name. Ko-tamago-tengutake. (小卵天狗咠).

Sect. Marginatae Schroet. 1. c. 679, 1889.

8. Amanita muscaria (Fr. ex L.) Qú́L.

Champ. Jura Vosg. 1, 25, 1872.

Agaricus muscarius L. Sp. Pl. 1172, 1753.

Amanita muscaria Pers. Syn. Fung. 253, 1801.

Agaricus (Amanita) muscarius Fr. Syst. Myc. 1, 16, 1821.

Venenarius muscarius MurRiLl, Mycologia 5, 75, 1913.

Hab. On the ground in woods, in solitary or gregarious. Sept.-Oct.

Distr. Hokkaido (Ishikari) \& Honshu. Europe, North America, Australia \& South Africa.

Jap. name. Beni-tengu-take, Ashi-taka-benitake, Aka-haitori-take, Ki-tengutake. (紅天狗苗, 脚高紅茸, 赤蠅探苗, 黄天狗苗). 
9. Amanita pantherina (Fr. ex DC.) QuÉL.

Camp. Jura Vosg. 1, 26, 1872.

Amanita umbrina Pers. Syn. Fung. 254, 1801.

Agaricus pantherinus DC. Fl. Fr. 6, 52, 1815.

Agaricus (Amanita) pantherinus Fr. Syst. Myc. 1, 16, 1821.

Hab. On the ground in woods, in solitary. Aug.-Oct.

Distr. Hokkaido (Ishikari \& Kushiro) \& Honshu. Europe \& North America. 豹茸)

Jap. name. T'engu-take, Haitori-take, Hyô-take.（天狗茸，蠅採咠，

\section{Amanita sepiacea Imai, sp. nov.}

Pileo 6-10 cm. lato, convexo, dein expanso, subumbrino vel subfuligineo, viscidulo, laevi, verrucis cinereis vel brunneis dense obtecto, margine laevi; carne alba, tenui, sapore odoreque nullo; lamellis vix liberis vel subadnatis, albis, utrimque attenuatis, confertis ; stipite 15-18 $\mathrm{cm}$. longo, $1-1.5 \mathrm{~cm}$. crasso, sursum attenuato, deorsum obovate bulboso, e cinereo fumoso vel avellaneo, fibrilloso vel fibrilloso-squamuloso, e solido deorsum farcto; annulo membranaceo, apicali, persistante, albo, striato, subtus avellaneo; volva adnata, 2-4 concentrice squamoso-marginata, evanida vel subpersistante; sporis globosis vel subglobosis, 8-10 $\mu$, hyalinis.

Hab. On the ground in woods, in solitary. Oct.

Distr. Hokkaido (Ishikari).

Jap. name. Tengu-take-modoki. (擬天狗咠).

The present species closely resembles Ananita Emilii RIEL. from which it is distinguishable in the pileus which is darker color even in the early stage and not striate on the margin, as well as in the darker colored stem.

The fungus also somewhat relates to a less rubescent form of Amanita rubescens, but it is easily distinguished by the marginate volva and the globose spores.

Sect. Incompletae Schroet. 1. c. 677, 1889.

11. Amanita rubescens (Fr. ex Pers.) QuéL.

Champ. Jura Vosg. 1, 27, 1872.

Agaricus rubens Scop. Fl. Carn. ed. 2, 2, 416, 1772.

Amanita rubescens Pers. Syn. Fung. 254, 1801.

Agaricus (Amanita) rubescens Fr. Syst. Myc. 1, 18, 1821.

Amanita rubens QuéL. Ench. Fung. 4, 1886.

Venenarius rubens MurriLL, Mycologia 5, 75, 1913. 
Hab. On the ground in woods, in solitary or scattered. Aug.-Sept.

Distr. Hokkaido (Ishikari \& Iburi) \& Honshu. Europe \& North America.

Jap. name. Gan-take, Irogawari-tengutake. (雁咠, 色變天狗咠).

12. Amanita spissa (Fr.) QuéL.

Champ. Jura Vosg. 1, 27, 1872.

Agaricus (Amanita) spissus Fr. Epicr. 9, 1836.

Hab. On the ground in woods, in solitary or gregarious. Sept.

Distr. Hokkaido (Ishikari) \& Honshu. Europe, North America \& Australia.

Jap. name. Hebi-kinoko. (蛇咠),

13. Amanita spissacea ImaI, sp. nov.

Pileo 6-10 cm. lato, convexo, dein expanso, e avellaneo atro-castaneo vel subfuligineo, verrucis atro-castaneis subfuligineisve dense concentrice obtecto ; carne alba, crassa, spissa, odore nullo, sapore blando ; lamellis liberis, albis, subconfertis, ventricosis, utrimque attenuatis ; stipite 10$15 \mathrm{~cm}$. longo, 1-2 cm. crasso, sursum attenuato, deorsum obovate bulboso, supra annulum subcinereo floccoso-squamosoque vel pulvere squamosoque, infra annulum squamoso, atro-castaneo, solido; annulo membranaceo, supero, albido, striato, subtus cinereo-albido floccosoque, margine atrocastaneo; volva pulvera, atro-castanea vel subfuliginea, vix concentrice obtecta, evanida; sporis globosis, $7-8 \mu$, hyalinis, apiculatis.

Hab. On the ground in woods, in solitary. Sept.

Distr. Hokkaido (Ishikari).

Jap. name. Hebikinoko-modoki. (擬蛇咠).

The present species closely allies to the preceding, Amanita spissa, from which it is easily distinguishable in the stem which is decorated by the hair-brown colored powdery scales, as well as in the globose spores. From Amanita valida, which resembles the present fungus the latter is easily distinguished by the globose spores and the stem enlarging to distinctly obovate-bulbous at the base.

\section{Amanita pulchella ImaI, sp. nov.}

Pileo 2.5-5 cm. lato, convexo, dein expanso, demum depresso, flavo, margine pallidiore striatuloque, viscido, glabro, fragmentis volvae luteae squamoso; carne alba, tenui, sapore odoreque nullo ; lamellis liberis, albis, demum flavulescentibus, confertis, ventricoso-fusiformibus ; stipite 8-10 $\mathrm{cm}$. longo, 4-7 mm. crasso, sursum leniter attenuato vel subaequali, deorsum non vel vix incrassato, albo vel albido, leniter luteo-pulverulente, 
farcto ; annulo membranaceo, supero, luteo, persistante; volva pulvera, lutea, evanida; sporis crasso-ellipsoideis, 7.5-10 $\times 5-6 \mu$, hyalinis.

Hab. On the ground in woods, in solitary or scattered. Aug.

Distr. Hokkaido (Ishikari).

Jap. name. Ko-kogane-tengu-take. (小黄金天狗咠).

The present species closely allies to Amanita Frostiana Pr. from which it is distinguishable in the stem which does not bulbously enlarge but hardly enlarges at the base, as well as by the broadly ellipsoidal spores. From Amanita flavoconia AtKins., which was described by AtKinson, Peck, KaUfFMan, etc., and was considered by MurRill as an identical species with A. Frostiana PK., the present fungus differs in the pileus striatulate on the margin and the stem not or hardly enlarged at the base.

15. Amanita flavipes Imar, sp. nov.

Pileo circa $6.5 \mathrm{~cm}$. lato, convexo, dein expanso, demum vix depresso, flavo-umbrino, margine pallidiore et leniter striatulo, viscidulo, laevi, glabro, fragmentis volvae luteae squamoso; carne tenuissima, odore nullo; lamellis albis vel flavidulis, liberis, confertis; stipite circa $10 \mathrm{~cm}$. longo, circa $1 \mathrm{~cm}$. crasso, sursum leniter attenuato vel subaequali, deorsum vix incrassato, supra annulum pulvero-squamuloso, flavulo, infra annulum pulvero-floccoso, luteo, cavo; annulo membranaceo, supero, flavulo, leniter striatulo, persistante ; volva pulvera, lutea, evanida; sporis subglobosis vel crasso-ellipsoideis, $8-9 \times 6-7 \mu$, hyalinis.

Hab. On the ground in woods, in solitary. Sept.

Distr. Hokkaido (Ishikari).

Jap. name. Kogane-tengu-take. (黄金天狗咠).

The present species closely resembles the preceding Amanita pulchella, from which it is distiguished by the clearly yellow colored stem.

\section{Amanitopsis Roze}

Bull. Soc. Bot. Fr. 23, 50, 1876.

Agaricus \& Vaginata NeEs v. Es. Syst. Pilze 191, 1817.

Vaginata S. F. Gray, Nat. Arr. Brit. Pl. 1, 601, 1821.

Amanitella EARLe, Bull. N. Y. Bot. Gard. 5, 449, 1909.

Amanita auct. p. p.

Sect. Volvatae Imar, sect. nov.

Volva vaginata. 
1. Amanitopsis vaginata (Fr. ex Bulc.) Roze

Bull. Soc. Bot. Fr. 23, 111, 1876.

Agaricus plumbeus Sch AefF. Fung. Bavar. pl. 85, 86, 4, Ind, 37, 1774. Agaricus vaginatus Bull. Champ. Fr. pl. 98, 1782.

Amanita vaginata Pers. Syn. Fung. 249, 1801.

Agaricus (Amanita) vaginatus Fr. Syst. Myc. 1, 14, 1821.

Agaricus (Amanita) vaginatus b. pil. griseo, livido. Fr. ibid. 14, 1821.

Amanitopsis plumbea Schroet. Pilze Schles. 1, 676, 1889.

Vaginata vaginata MurriLL, Mycologia 3, 80, 1911.

Vaginata plumbea Murnilu, ibid. 5, 82, 1913.

Amanita vaginata subsp. plumbea Konr. et MaUb. Icon. Select. Fung. 1, 33, 1924-30.

Hab. On the ground in woods, in solitary or scattered. Sept.-Oct.

Distr. Hokkaido (Ishikari \& Iburi) \& Honshu. Europe, North America \& Australia.

Jap. name. Tsuru-take. (鶴茸).

2. Amanitopsis albida (Fr.) ImaI, comb. nov.

Agaricus (Amanita) vaginatus a pileo albida Fr. Syst. Myc. 1, 14, 1821.

Agaricus (Amanita) vaginatus var. albida FR. Epicr. 11, 1836.

Amanita vaginata var. alba GiLl. Hymén. Fr. 51, 1874.

Hab. On the ground in woods, in solitary. Aug.

Distr. Hokkaido (Ishikari) \& Honshu. Europe, North America \& Australia.

Jap. name. Yuki-tsurutake (?=Shiro-tsurutake). (雪鶴苗).

Amanita nivalis Grev. has been treated by many as a white form of Amanitopsis vaginata, but $\mathrm{REA}_{\mathrm{EA}}$ has described that the spores of Amanitopsis nivalis (GREv.) REA are oblong elliptic, $11-12 \times 9 \mu$, with a large central gutta. The spores of our fungus are globose, 10-12.5 $\mu$, containing a large central gutta.

3. Amanitopsis fulva (Fr. ex Schaef F.) W. G. Smith Syn. Brit. Basid. 18, 1908.

Agaricus fulvus Schaeff. Fung. Bavar. pl. 95, 4, Ind. 41, 1774.

Agaricus trilobus Boct. Hist. Fung. 1, 38, pl. 38, f. 2, 1788.

Agaricus (Amanita) vaginatus e. p. fulvo, Fr. Syst. Myc. 1, 14, 1821.

Ayaricus (Amanita) vaginatus var. fulva Fr. Hym. Eur. 27, 1874.

Amanita vaginata var. fulva GILL. Hymén. Fr. 51, 1874.

Amanita viginata subsp. fulva Kovr. et MAUBL. Icon. Select. Fung. 1, 33, 1924-30. 
Hab. On the ground in woods, in solitary. Sept.

Distr. Hokkaido (Ishikari) \& Honshu. Europe \& North America. Jap. name. Kaba-iro-tsurutake. (樺色鶴革).

\section{Amanitopsis avellaneosquamosa ImaI, sp. nov.}

Pileo 5-10 cm. lato, convexo, dein expanso, demum depresso, sicco, avellaneo-squamoso, margine striato ; carne alba, crassa, odore saporeque nullo ; lamellis albis, demum flavulescentibus, liberis, confertis, vix ventricosis ; stipite $9-17 \mathrm{~cm}$. longo, $0.6-2 \mathrm{~cm}$. crasso, aequali vel sursum leniter attenuato, squamoso, concolori vel leniter pallidiore, e farcto cavo; volva membranacea, ampla, vaginali, albida, persistante ; sporis ellipsoideis, 9$10.5 \times 5-6 \mu$, hyalinis.

Hab. On the ground in woods, in solitary or scattered. Aug.

Distr. Hokkaido (Ishikari).

Jap. name. Aku-iro-uroko-tsurutake. (灰色鱗鶴茸).

The present species somewhat resembles the preceding Amanitopsis fulva from which it is easily distinguishable in the clearly scaly pileus and the ellipsoidal spores.

\section{Amanitopsis clarisquamosa Imai, sp. nov.}

Pileo 4-6 cm. lato, hemisphaerico vel convexo, dein expanso, sicco, centro laevi, albido, flavulo vel brunneolo, margine albido, clare fibrillososquamoso, subisabellino ; carne albido, sapore odoreque nullo; lamellis liberis, albis, confertis, ventricosis ; stipite $6-10 \mathrm{~cm}$. longo, $0.8-1.2 \mathrm{~cm}$. crasso, sursum leniter attenuato, albido vel brunneolo, dense pulvero- vel floccoso-squamoso, subincarnato, solido vel farcto; volva ampla, membranacea, vaginali; lobata, brunneola vel albida, persistante; sporis ellipsoideis, $10-15 \times 5-6 \mu$, hyalinis.

Hab. On the ground in woods, in solitary or scattered. Oct.

Distr. Hokkaido (Ishikari).

Jap. name. Shiro-uroko-tsurutake. (白鱗鶴茸).

The present fungus resembles Amanitopsis volvata (PK.) SACC. from which it is easily distinguished by the evidently scaly pileus and stipe, as well as by the more slender spores.

Sect. pulverntme IMaI, sect. nov.

Volva pulveracea.

6. Amanitopsis farinosa (Schw.) Atkins.

Stud. Amer. Fungi 76, 1900.

Amanita farinosa Schw. Schr. Nat. Ges. Leipzig 1, 79, 1822. 
Agaricus (Amanita) farinosus Fr. Epicr. 11, 1836.

Vaginata furinosa MurnILL, Mycologia 4, 3, 1912. Oct.

Hab. On the grassy ground in woods, in solitary or gregarious July-

Distr. Hokkaido (Ishikari). North America.

Jap. name. Hime-konakaburi-tsurutake. (姬被粉鶴咠).

Volvaria (Fr.) QuéL.

Champ. Jura Vosg. 1, 72, 1872.

Agaricus \& Volvaria Fr. Syst. Myc. 1, 277, 1821.

Pseudofarinaceus Earle, Bull. N. Y. Bot. Gard. 5, 449, 1909.

Volvariopsis MurriLL, Mycologia 3, 280, 1911.

1. Volvaria bombjcina (Fr. ex SchaefF.) QUÉL.

Champ. Jura Vosg. 1, 72, 1872.

Agaricus bombycinus Sch AEfF. Fung. Bavar. pl. 98, 4, Ind. 42, 1774.

Aguricus (Volvaria) bombycinus Fr. Syst. Myc. 1, 277, 1821.

Volvariopsis bombycina MurriLL, Mycologia 3, 281, 1911.

Hab. On decayed wood or on bark of living wood, in solitary. JulySept.

Distr. Hokkaido (Ishikari) \& Honshu. Siberia, Europe, North America, Australia \& Africa.

Jap. name. Kinu-ô-fukuro-take. (絹大袋苗).

2. Volvaria volvacea (Fr. ex BuLL.) QuéL.

Ench. Fung. 54, 1886.

Agaricus volvaceus Bulc. Champ. Fr. pl. 262, 1785.

Agaricus virgatus Pers. Tent. Disp. Fung. 18, 1797.

Amanita virgaia Pers. Syn. Fung. 249, 1801.

Agaricus (Volvaria) volvaceus Fr. Syst. Myc. 1, 278, 1821.

Volvaria virgata QuÉL. Champ. Jura Vosg. 2, 12, 1873.

Volvariopsis volvacea MurRILL, North Amer. Fl. 10, 144, 1917.

Hab. On the ground in woods, in solitary or scattered. Sept.

Distr. Hokkaido (Ishikari) \& Honshu. Ceylon, Europe \& North America.

Jap. name. Fukuro-take. (袋茸).

3. Volvaria speciosa (Fr.) GILL.

Hymén. Fr. 388, 1874. 
Agaricus (Volvaria) speciosus Fr. Syst. Myc. 1, 278, 1821.

Volvariopsis speciosa Murrill, North Amer. Fl. 10, 143, 1917.

Hab. On the ground in woods, in solitary. June-July.

Distr. Hokkaido (Ishikari). Europe, Australia \& North America. Jap. nane. Shiro-fukurotake. (白袋茸).

4. Volvaria gloiocephala (Fr. ex DC.) GILL.

Hymén. Fr. 387, 1874.

Agaricus gloiocephalus DC. Fl. Fr. 6, 52, 1815.

Agaricus (Volvaria) gloiocephalus. Fr. Syst. Myc. 1, 278, 1821.

Volvariopsis gloiocephala Murrilu, North Amer. Fl. 10, 144, 1917.

Hab. On the ground in woods, in solitary. July-Oct.

Distr. Hokkaido (Ishikari). Europe.

Jap. name. Ô-fukuro-take. (大袋茸).

Botanical Institute,

Faculty of Agriculture,

Hokkaido Imperial University,

Sapporo, Japan 